\title{
Effect of Upper- and Lower-Level Baroclinicity on the Persistence of the Leading Mode of Midlatitude Jet Variability
}

\author{
LOḮC ROBERT AND GWENDAL RIVIÈRE \\ LMD/IPSL, École Normale Supérieure, PSL Research University, École Polytechnique, \\ Université Paris Saclay, Sorbonne Universités, CNRS, Paris, France \\ FRANCIS CODRON \\ LOCEAN/IPSL, Sorbonne Universités, CNRS, Paris, France
}

(Manuscript received 9 January 2018, in final form 12 September 2018)

\begin{abstract}
The sensitivity of the variability of an eddy-driven jet to the upper- and lower-level baroclinicity of the mean state is analyzed using a three-level quasigeostrophic model on the sphere. The model is forced by a relaxation in temperature to a steady, zonally symmetric profile with varying latitude and intensity of the maximum baroclinicity. The leading EOF of the zonally and vertically averaged zonal wind is characterized by a meridional shift of the eddy-driven jet. While changes in the upper-level baroclinicity have no significant impact on the persistence of this leading EOF, an increase in lower-level baroclinicity leads to a reduced persistence. For small lower-level baroclinicity, the leading EOF follows a classical zonal index regime, for which the meridional excursions of the zonal wind anomalies are maintained by a strong positive eddy feedback. For strong lower-level baroclinicity, the jet enters a poleward-propagation regime, for which the eddy forcing continuously acts to push the jet poleward and prevents its maintenance at a fixed latitude. The enhanced poleward propagation when the lower-level baroclinicity increases is interpreted as resulting from the broader and weaker potential vorticity gradient that enables the waves to propagate equatorward and facilitates the poleward migration of the critical latitude. Finally, the decrease in the persistence of the leading EOF as the lower-level baroclinicity increases is shown not to result from the impact of changes in the mean climatological jet latitude.
\end{abstract}

\section{Introduction}

Eddy-driven jets and storm tracks have a key role in midlatitude surface weather, and it is important to understand how they might respond to climate change. Results from the Coupled Model Intercomparison Project (CMIP) experiments show a robust poleward shift of the jet in the Southern Hemisphere (Kidston and Gerber 2010), but the picture is more complicated in the Northern Hemisphere ocean basins (Barnes and Polvani 2013; Simpson et al. 2014; Vallis et al. 2015). In particular, there are competing influences of the local warming maxima in the upper tropical troposphere (Seager et al. 2003; Lu et al. 2008) and near the surface in the Arctic (Oudar et al. 2017), which change the meridional temperature gradient in opposite directions at different levels in the vertical and therefore have

\footnotetext{
Corresponding author: Loïc Robert, lrobert@lmd.ens.fr
}

opposite effects in terms of jet shifts (Butler et al. 2010; Rivière 2011; Chavaillaz et al. 2013; Harvey et al. 2014; Shaw et al. 2016).

A change of the mean jet latitude can change its variability; as the mean jet moves poleward, its leading mode of variability - usually characterized by a latitudinal wobbling_often becomes less persistent, possibly because of a weaker positive eddy feedback (Barnes et al. 2010; Kidston and Gerber 2010; Arakelian and Codron 2012, see Fig. 5e therein), even if some studies with comprehensive models suggest that this relationship may not be as robust as initially thought (Simpson et al. 2013a; Simpson and Polvani 2016). Moreover, the dominant mode of variability may change its nature for high-latitude jets and become characterized more by a pulse in amplitude than by a latitudinal shift (Barnes and Hartmann 2011). The jet variability seems also strongly sensitive to the latitude of wave excitation, either the latitude of stochastic stirring in a 
barotropic model (Barnes et al. 2010) or that of oceanic fronts in aquaplanet general circulation model (GCM) experiments (Michel and Rivière 2014; Ogawa et al. 2016). In these studies, the persistence of the leading mode of variability decreases as the wave excitation zone is shifted poleward, in agreement with the sensitivity to the jet latitude.

The impacts on the jet variability of changes in the mean temperature gradient or baroclinicity have been comparatively less studied. Son and Lee $(2005,2006)$ performed sensitivity numerical experiments in a dry GCM by varying the tropical heating and high-latitude cooling rates of the radiative equilibrium temperature profile used to force their model. The intensity of the tropical heating controlled the strength of the subtropical jet while the high-latitude cooling largely determined the width and strength of the midlatitude baroclinic zone. For large tropical heating and small polar cooling, a single jet appeared, and the leading mode was a latitudinal wobbling of the jet (also referred to as the zonal index regime). In the opposite range of the parameter space (small tropical heating and large polar cooling), a double-jet structure emerged at upper levels, and zonal wind anomalies tended to propagate poleward (also referred to as the poleward-propagation regime). The interpretation mainly relied on the shape of the time-mean potential vorticity gradient (Lee et al. 2007; Son et al. 2008). When it is strong and sharp as in the first case (zonal index), waves are meridionally trapped and mainly converge momentum into the jet core, leading to a strong positive eddy feedback. When it is weak and broad as in the second (propagating) case, waves propagate more easily equatorward and push the jet poleward. Similar arguments will be used in the present study when analyzing the sensitivity of the jet variability to the strength of the baroclinicity using a quasigeostrophic model on the sphere. It is important to underline that our numerical setup significantly differs from the previously cited studies as the subtropical jet is weak in our quasigeostrophic framework. Also, the originality of the present paper lies in the distinction made between the effects of changes in lower-level and upper-level baroclinicity. Changes in the vertical structure of the baroclinicity are known to have an effect on jet position (Butler et al. 2010; Rivière 2011) and eddy intensity (Held and O'Brien 1992; Yuval and Kaspi 2016), but its effect on the jet variability has not apparently been studied so far.

The idealized quasigeostrophic model used in the present study has three vertical levels, which is the minimal framework to separate the baroclinicity into lower- and upper-level components. This model is thus well suited to study in particular the effect of opposite tendencies in lower- and upper-level temperature gradients, as occurs in global warming scenarios (Held and O'Brien 1992; Rivière 2011). The model is described in section 2, together with the different experiments. The results describing the changes in the jet variability and eddy feedbacks when modifying the lower- and upperlevel baroclinicity are presented in section 3 , together with a dynamical interpretation and sensitivity experiments to check the robustness of the results. Concluding remarks are provided in section 4.

\section{Model and diagnostics}

\section{a. Numerical model}

The model used is the dry quasigeostrophic model on the sphere of Marshall and Molteni (1993) at T42 resolution. It has three pressure levels in the vertical at 200, 500 , and $800 \mathrm{hPa}$. The model is governed by the following equations for the quasigeostrophic potential vorticity $(\mathrm{PV}) q_{i}$ at levels $i=1,2,3$ :

$$
\begin{aligned}
& \frac{\partial q_{i}}{\partial t}+J\left(\psi_{i}, q_{i}\right)=S_{i}-D_{i}, \\
& q_{i}=f+\Delta \psi_{i}-\left(\frac{\psi_{i}-\psi_{i+1}}{R_{i}^{2}}-\frac{\psi_{i-1}-\psi_{i}}{R_{i-1}^{2}}\right),
\end{aligned}
$$

where $\psi_{i}$ is the streamfunction $\left(\psi_{0}=\psi_{1}\right.$ and $\left.\psi_{4}=\psi_{3}\right), R_{i}$ is the deformation radius between levels $i$ and $i+1, S_{i}$ and $D_{i}$ are the source and dissipative terms, $\Delta$ denotes the Laplacian operator, and $f=2 \Omega \sin (\varphi)$ is the Coriolis parameter. The deformation radii are set to $R_{1}=600 \mathrm{~km}$ and $R_{2}=400 \mathrm{~km}$, as in Rivière (2009) and Robert et al. (2017, hereafter RRC17).

Source and dissipative terms are the sum of three distinct contributions that can be expressed as

$$
\begin{aligned}
S_{1}-D_{1}= & -c_{H} \nabla^{8}\left(q_{1}-f\right)+\frac{1}{\tau_{R_{1}}} \frac{\psi_{1}-\psi_{2}-\tilde{\psi}_{1}+\tilde{\psi}_{2}}{R_{1}^{2}}, \\
S_{2}-D_{2}= & -c_{H} \nabla^{8}\left(q_{2}-f\right)-\frac{1}{\tau_{R_{1}}} \frac{\psi_{1}-\psi_{2}-\tilde{\psi}_{1}+\tilde{\psi}_{2}}{R_{1}^{2}} \\
& +\frac{1}{\tau_{R_{2}}} \frac{\psi_{2}-\psi_{3}-\tilde{\psi}_{2}+\tilde{\psi}_{3}}{R_{2}^{2}}, \\
S_{3}-D_{3}= & -c_{H} \nabla^{8}\left(q_{3}-f\right) \\
& -\frac{1}{\tau_{R_{2}}} \frac{\psi_{2}-\psi_{3}-\tilde{\psi}_{2}+\tilde{\psi}_{3}}{R_{2}^{2}}-\frac{1}{\tau_{E}} \nabla^{2} \psi_{3} .
\end{aligned}
$$

The first term on the right-hand side (rhs) of the equation represents a scale-selective horizontal diffusion 
such that the damping time scale of the shorter waves at T42 truncation is 0.02 days. The next term on the rhs represents a Newtonian relaxation in temperature toward a fixed profile (denoted with a tilde) using two time scales at the two interfaces. Finally, a linear drag with a time scale of $\tau_{E}=3$ days is applied to the third level.

The restoration temperature profile is zonally symmetric and in thermal wind balance with a Gaussian zonal jet given by

$$
\tilde{u}_{i}(\lambda, \varphi)=U_{i} \exp \left[-\frac{\left(\varphi-\varphi_{0}\right)^{2}}{d \varphi^{2}}\right],
$$

where $\varphi_{0}=30^{\circ} \mathrm{N}$ and $d \varphi=20^{\circ}$ are the control values of the mean position and width of the jet. Control values of the restoration wind amplitude $U_{i}$ are set to $U_{1}=U_{0}$, $U_{2}=0.5 U_{0}$, and $U_{3}=0.2 U_{0}$, where $U_{0}=50 \mathrm{~m} \mathrm{~s}^{-1}$. In the rest of the paper, we define the baroclinicity by the vertical shear of the zonal wind divided by the radius of deformation at the interface because the static stability is a constant at each interface. The upper- (lower-) level baroclinicity refers to $\left(u_{1}-u_{2}\right) / R_{1}\left[\left(u_{2}-u_{3}\right) / R_{2}\right]$, and its restoration counterpart is $\left(\tilde{u}_{1}-\tilde{u}_{2}\right) / R_{1}\left[\left(\tilde{u}_{2}-\tilde{u}_{3}\right) / R_{2}\right]$. The simulations are 15 years long to ensure significant results, and the first year is discarded before the analysis, as in RRC17.

\section{b. Simulations}

Different series of simulations have been performed to investigate the impact of changes of the upper- and lower-level baroclinicity. These series are shown in Table 1. Experiments in which the upper-level baroclinicity is varied (denoted as UB) are obtained by fixing the equilibrium lower-level wind amplitude $U_{2}$ and $U_{3}$ to their respective control values and varying $U_{1}$ from $0.8 U_{0}$ to $1.2 U_{0}$, corresponding to an upper-level baroclinicity factor $\left(U_{1}-U_{2}\right) / U_{0}$ varying from 0.3 to 0.7 . That means that there is a factor of about 2.3 between the smallest and greatest values of the upper-level vertical shear of the restoration zonal wind. Similarly, experiments where the lower-level baroclinicity changes are obtained by fixing $U_{1}$ and $U_{2}$ and varying $U_{3}$ from $0.3 U_{0}$ to $0.1 U_{0}$. This corresponds to a baroclinicity factor $\left(U_{2}-U_{3}\right) / U_{0}$ varying from 0.2 to 0.4 and a lower-level vertical shear varying by a factor of 2 .

The study is mainly focused on sensitivity experiments for which the relaxation time scales are set to $\tau_{R_{1}}=$ $\tau_{R_{2}}=25$ days. This enables a systematic comparison between the effects of changes in upper- and lower-level baroclinicity as the relaxation time scales are the same at the two interfaces. These series are hereafter denoted as UB25 and LB25. Another set of sensitivity experiments
TABLE 1. Sensitivity experiment description table.

\begin{tabular}{lcccccc}
\hline \hline Name & $\tau_{R_{12}}$ & $\tau_{R_{23}}$ & Parameter & Minimum & Maximum & Step \\
\hline JP25 & 25 & 25 & $\varphi_{0}$ & $20^{\circ} \mathrm{N}$ & $40^{\circ} \mathrm{N}$ & $2^{\circ}$ \\
UB25 & 25 & 25 & $U_{1} / U_{0}$ & 0.8 & 1.2 & 0.1 \\
LB25 & 25 & 25 & $U_{3} / U_{0}$ & 0.1 & 0.3 & 0.02 \\
UB40 & 40 & 15 & $U_{1} / U_{0}$ & 0.8 & 1.2 & 0.1 \\
LB40 & 40 & 15 & $U_{3} / U_{0}$ & 0.1 & 0.3 & 0.02 \\
\hline
\end{tabular}

is made with different time scales at the upper and lower interfaces $\left(\tau_{R_{1}}=40\right.$ days and $\tau_{R_{2}}=15$ days), as used in RRC17, hereafter denoted as UB40 and LB40. Finally, a series of simulation JP25 with fixed baroclinicity but changes in the jet position $\varphi_{0}$ is run to help assess the respective impacts of changes in the baroclinicity and jet latitude.

\section{Results}

\section{a. Time-mean versus restoration baroclinicity}

Changes in the baroclinicity of the restoration temperature field do not necessarily induce the same changes in the simulated climatology because of the dynamical adjustment. Figure 1 shows the climatological upper- and lower-level baroclinicity fields obtained for the UB25 and LB25 experiments. An increase in the upper- or lower-level restoration baroclinicity indeed leads to an increase of the corresponding climatological mean baroclinicity, as shown in Figs. 1a and 1d. We note that the upper-level baroclinicity maxima differ by a factor of 2 between the extreme cases in UB25 (Fig. 1a), which is a bit less than the range of the restoration baroclinicity. There is however little impact on the lower-level baroclinicity, with only a slight decrease and shift toward the pole.

For the LB25 series, the climatological mean lowerlevel baroclinicity also fluctuates over a smaller range of maxima than the restoration baroclinicity (Fig. 1b), but unlike the UB25 case, the increase in the lower-level baroclinicity in LB25 simulations leads to a decrease in the upper-level one. This change in upper-level baroclinicity is because the upper-level wind does not change as the lower-level baroclinicity is increased, while the mid- and lower-level winds increase (not shown). The increase in both the lower- and midlevel winds can be interpreted as follows. When the lowerlevel baroclinicity is increased, eddy energy increases, but as the dissipation is acting at the low level only, the low-level wind must increase. This also leads to an increase in the midlevel wind because the forcing is such that the baroclinicity at the interface between the lowand midlevel increases. These opposite changes in the two baroclinicities need to be kept in mind while 

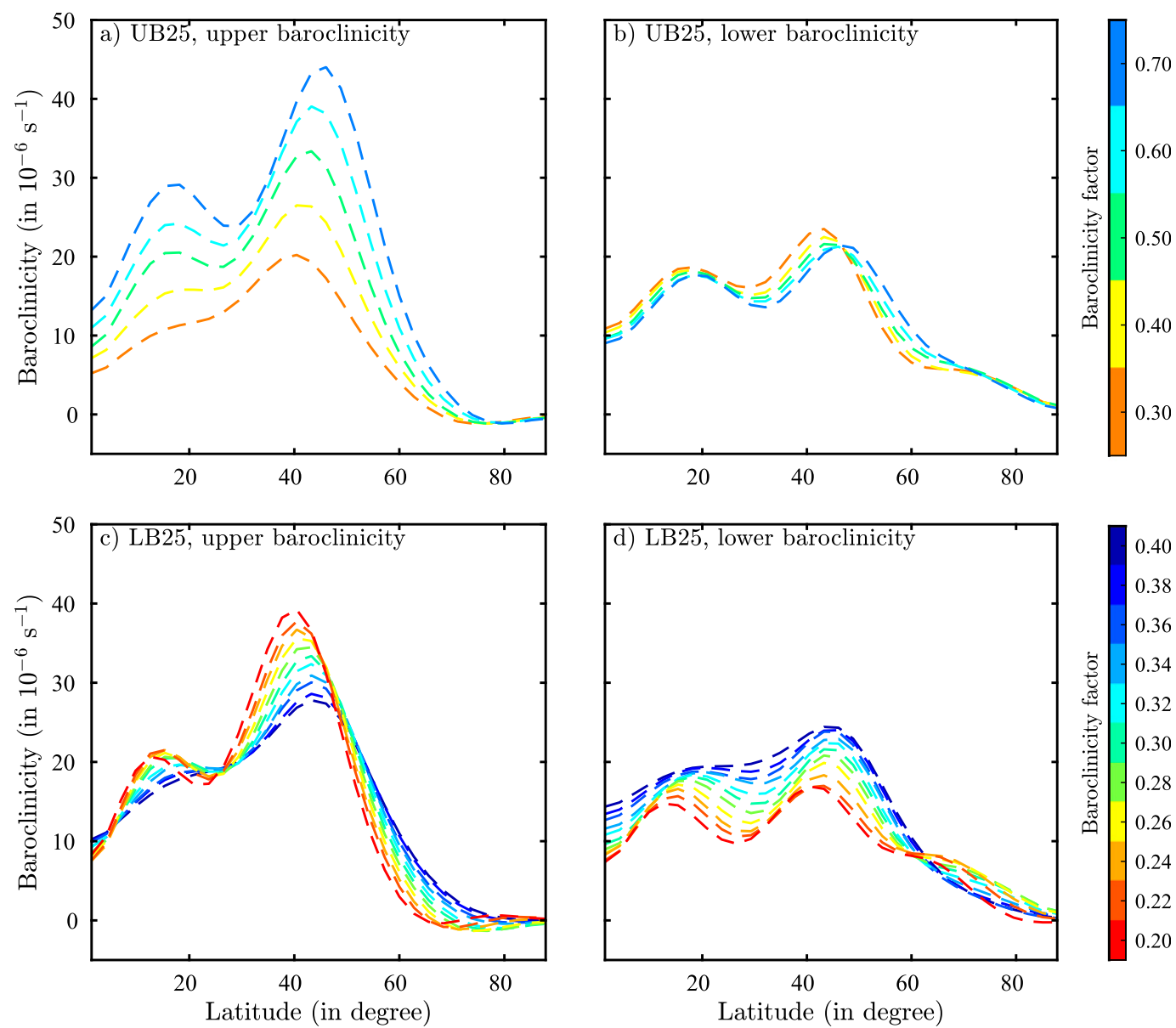

FIG. 1. Climatological mean of (a),(c) upper- and (b),(d) lower-level baroclinicity, respectively, $\left(U_{1}-U_{2}\right)$ and $\left(U_{2}-U_{3}\right)$, for (a),(b) UB25 and (c),(d) LB25. The baroclinicity factors correspond to $\left(U_{1}-U_{2}\right) / U_{0}$ and $\left(U_{2}-U_{3}\right) / U_{0}$, respectively.

interpreting the results. Similar results are obtained for UB40 and LB40 (not shown).

\section{b. Persistence of the leading mode and eddy feedbacks}

Following Lorenz and Hartmann (2001) and RRC17, empirical orthogonal functions (EOFs) of the zonally and vertically averaged zonal wind are computed for each simulation of UB25 and LB25. The autocorrelation function of the first principal component (PC1) of the leading EOF (EOF1) of each experiment is plotted in Fig. 2. As in RRC17, the autocorrelation has systematically a shoulder shape marked by a fast decay rate during the first few days followed by a slower decay rate at longer lags. On average, the $e$-folding time is around 20 days, which is of the same order of the time scales of the Southern Hemisphere annular mode in comprehensive models as they vary between 15 and 30 days during summer (Kidston and Gerber 2010;
Arakelian and Codron 2012). It is however larger than the observed ones, which are closer to an $e$-folding time of 10 days (Simpson et al. 2013b; Simpson and Polvani 2016), as is often the case in comprehensive and idealized models. A change in upper-level baroclinicity does not seem to impact the EOF1 persistence very much (Fig. 2a); however, an increase in the lower-level baroclinicity causes a clear decrease in persistence (Fig. 2b). After 20 days, the PC1 autocorrelation for strong lower-level baroclinicity $\left(U_{2}-U_{3} \geq\right.$ $0.34 U_{0}$ ) drops to 0.25 on average, whereas for weak lower-level baroclinicity $\left(U_{2}-U_{3} \leq 0.26 U_{0}\right)$, it remains above 0.5 .

The tendency of the vertically and zonally averaged zonal wind $\langle[u]\rangle$ satisfies the following equation (RRC17):

$$
\frac{\partial\langle[u]\rangle}{\partial t}=-\frac{1}{a \cos ^{2} \varphi} \frac{\partial}{\partial \varphi}\left(\left\langle\left[u^{*} v^{*}\right]\right\rangle \cos ^{2} \varphi\right)-\frac{\left[u_{3}\right]}{3 \tau_{E}},
$$



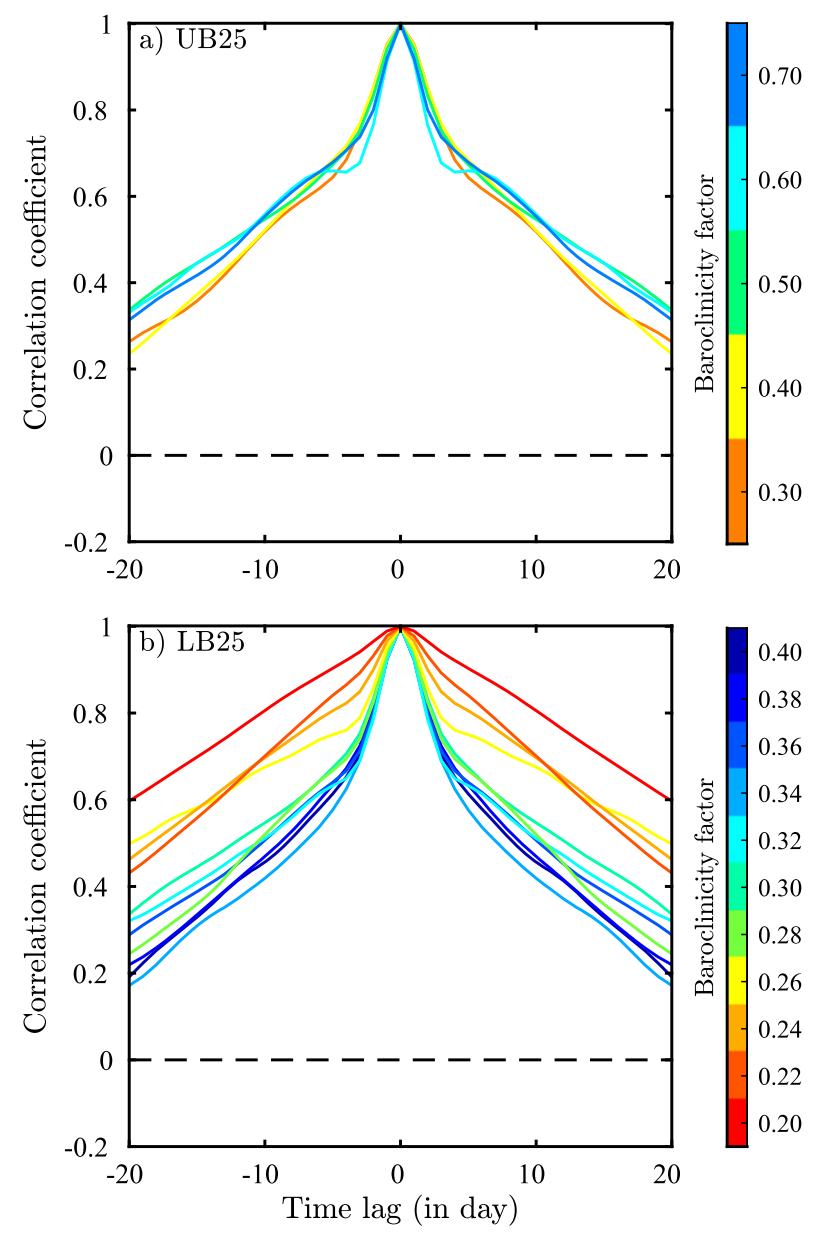

FIG. 2. Autocorrelation function of PC1 for (a) UB25 and (b) LB25 for which the baroclinicity factors are $\left(U_{1}-U_{2}\right) / U_{0}$ and $\left(U_{2}-U_{3}\right) / U_{0}$, respectively.

where $u_{3}$ is the zonal wind in the lower level of the model, the operators $\langle\cdot\rangle$ and $[\cdot]$ correspond to the vertical and zonal average, respectively, and $u^{*}=$ $u-[u]$ and $v^{*}=v-[v]$ are departures of the zonal and meridional wind from their zonal mean. The factor $1 / 3$ in the second term on the right-hand side of Eq. (3) is due to the vertical integration of the momentum equation and the fact that the Ekman dissipation is only acting in the lowest level. By projecting each term of Eq. (3) onto EOF1, we get the tendency equation for PC1:

$$
\frac{d \mathrm{PC} 1}{d t}=m+d
$$

where $m$ is the eddy momentum forcing (i.e., the projection of the eddy momentum flux convergence) and $d$ is the projection of the surface drag. To get growth rates at a given time $t$, we compute the time-lag cross correlation between PC1 and each term of Eq. (4), then we divide them by the autocorrelation function of PC1, $A_{1}\left(t_{0}\right)=\sum_{t} \operatorname{PC} 1(t) \mathrm{PC} 1\left(t_{0}+t\right)$ :

$$
\frac{1}{A_{1}} \frac{d A_{1}}{d t}=\frac{1}{\tau_{m}}+\frac{1}{\tau_{d}}
$$

where

$$
\begin{aligned}
\tau_{m}^{-1}\left(t_{0}\right) & =\frac{\sum_{t} \mathrm{PC} 1(t) m\left(t_{0}+t\right)}{A_{1}\left(t_{0}\right)}, \\
\tau_{d}^{-1}\left(t_{0}\right) & =\frac{\sum_{t} \mathrm{PC} 1(t) d\left(t_{0}+t\right)}{A_{1}\left(t_{0}\right)} .
\end{aligned}
$$

The left-hand side of Eq. (5), which is the instantaneous rate of change of the PC1 autocorrelation, is driven by two distinct contributions. On one hand, $\tau_{m}^{-1}$ corresponds to the forcing due to momentum flux convergence. On the other hand, $\tau_{d}^{-1}$ corresponds to the effect of the drag. The benefit of Eq. (5) is that $\tau_{d}^{-1}$ is almost constant. Indeed, $\left[u_{3}\right]$ is roughly proportional to $\langle[u]\rangle$, so we expect $d$ to evolve in line with PC1. Therefore, changes in the instantaneous rate of change of the PC1 autocorrelation should mainly be caused by changes in the eddy momentum forcing $\tau_{m}^{-1}$. Figure 3 shows $\tau_{m}^{-1}$ as a function of time lag and the baroclinicity factors $\left(U_{1}-U_{2}\right) / U_{0}$ and $\left(U_{2}-U_{3}\right) / U_{0}$. In general, this quantity is very similar to the time-lag cross covariance between PC1 and the eddy momentum forcing [i.e., $\left.\sum_{t} \mathrm{PC} 1(t) m\left(t_{0}+t\right)\right]$. It exhibits strong positive values at short negative lags that correspond to the triggering of the event by a strong projection of the convergence of eddy momentum onto the EOF. Then, at short positive lags, it has negative values in most cases. This was shown to come mainly from the negative feedback exerted by planetary waves when they reflect on the flanks of the jet (Rivière et al. 2016; RRC17). At longer positive lags, it usually reaches positive values because of a positive synoptic eddy feedback (e.g., Lorenz and Hartmann 2001, 2003; Zurita-Gotor 2014; RRC17). Even though the eddy momentum contribution to $A_{\mathrm{PC} 1}$ rate of change follows these different steps in most cases, differences among the various cases exist and are worth analyzing. As expected from Fig. 2, the values of the eddy momentum contribution show almost no monotonic or consistent changes when modifying the upperlevel baroclinicity (Fig. 3a). One notable feature is the strong oscillation for $U_{1}-U_{2}=0.6 U_{0}$, which is probably due to successive wave reflections (Rivière et al. 2016; RRC17). Conversely, Fig. 3b shows a gradual increase in the eddy momentum contribution at positive lags when the lower-level baroclinicity is decreased. At short 

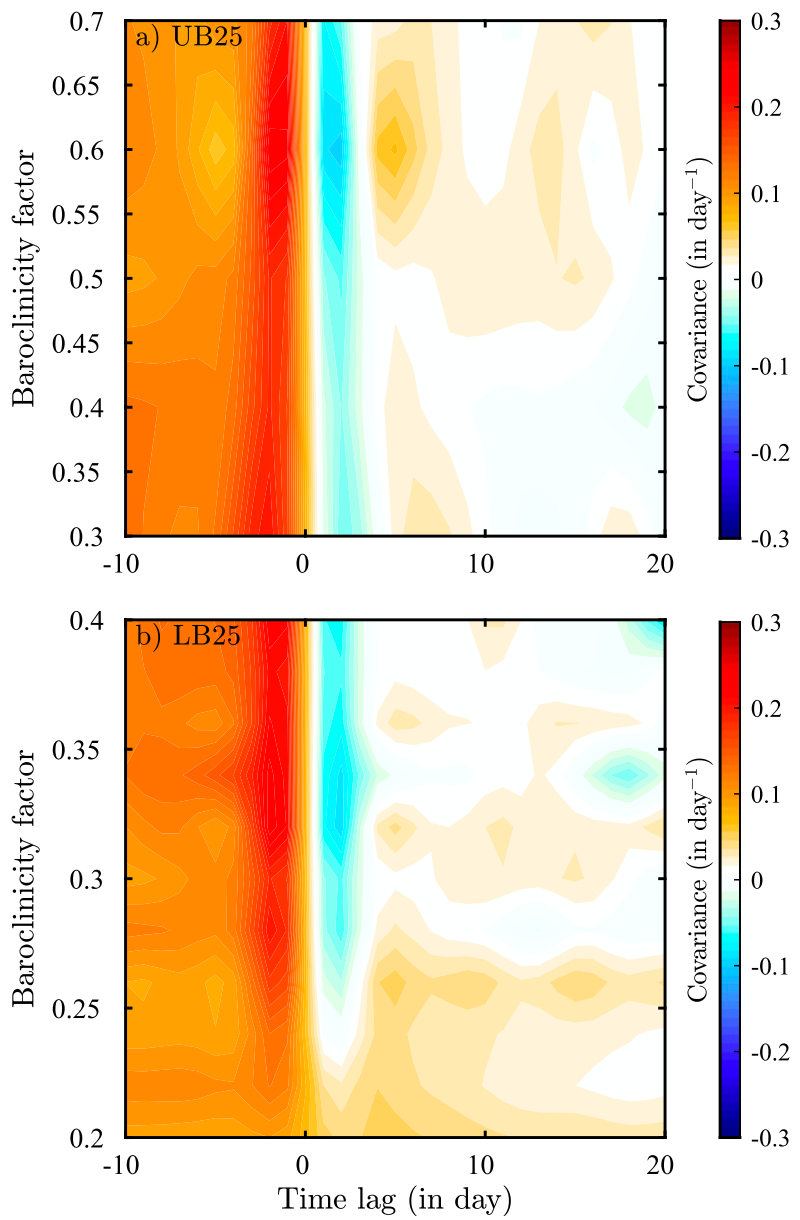

FIG. 3. Eddy momentum contribution to the rate of change of the PC1 autocorrelation function $\left[\tau_{m}^{-1}\right.$ of Eq. (5)], as a function of lag and baroclinicity factors for (a) UB25 and (b) LB25. The baroclinicity factors in (a) and (b) correspond to $\left(U_{1}-U_{2}\right) / U_{0}$ and $\left(U_{2}-U_{3}\right) / U_{0}$, respectively.

positive lags ( +2 to +3 days), the negative values found for large baroclinicity disappear and become positive for small baroclinicity. At longer positive lags $(+5$ to +20 days), the eddy momentum contribution is near zero for large baroclinicity and becomes positive for small baroclinicity.

Figure 4 compares each term of Eq. (5) for two extreme values of the lower-level baroclinicity. As expected, the contribution of the drag is constant and similar for both simulations. For weak lower-level baroclinicity (Fig. 4a), the eddy momentum contribution is significantly positive at all positive lags. But for strong lower-level baroclinicity (Fig. 4b), it is first negative at short positive lags, then becomes slightly positive between lag +5 and +12 days, and finally decreases and becomes again negative after lag +16 days. Hence, at all positive lags, the eddy momentum contribution $\tau_{m}^{-1}$ is stronger in the weak baroclinicity case.
Since the seminal work of Lorenz and Hartmann (2001), the positive correlation between $m$ and PC1 at long positive lags (greater than 5 days) is considered as the signature of a positive synoptic eddy feedback. Following this interpretation, the first main conclusion from Figs. 3 and 4 is that this positive feedback is weaker and less durable for stronger lower-level baroclinicity. The second conclusion is that, at short positive lags (less than 5 days), the negative feedback exerted by planetary waves as revealed in Rivière et al. (2016) and RRC17 becomes less effective for weak baroclinicity. These two changes go in the same direction, namely, a decrease in persistence of the leading mode of jet variability with increased lower-level baroclinicity. The next section will be dedicated to investigating the underlying causes.

\section{c. Dynamical interpretation}

The aim of this section is to interpret the clear drop in PC1 persistence when the lower-level baroclinicity is increased. Figure 5 shows the lagged regressions of the momentum flux convergence and zonal wind anomalies on PC1 for weak and strong lower-level baroclinicity. We first note that wind anomalies have more than three times higher amplitudes for stronger baroclinicity. This is logical as the lower-level baroclinicity largely determines the eddy growth rate (Lindzen and Farrell 1980; Hoskins and Valdes 1990), so the higher amplitude of eddies for stronger baroclinicity leads to stronger momentum flux convergence and jet fluctuations. More interestingly, zonal wind anomalies propagate poleward for strong lower-level baroclinicity while they stay more or less at the same latitude for weak lower-level baroclinicity. For example, for the weak lower-level baroclinicity simulation, the maximum of negative wind anomalies (dashed contours on Fig. 5a) is around $32^{\circ} \mathrm{N}$ for a lag of -10 days and around $34^{\circ} \mathrm{N}$ for a lag of +20 days. In contrast, for a strong lower-level baroclinicity, the same anomalies peaks at $35^{\circ} \mathrm{N}$ for a lag of -10 days and around $44^{\circ} \mathrm{N}$ for a lag of +20 days. This difference can explain the eddy momentum contribution to $A_{1}$ rate of change seen in Fig. 4b. Indeed, the poleward-shifted zonal wind anomalies of Fig. 5b are due to poleward-shifted eddy momentum convergence patterns after lag +5 days, which end up becoming orthogonal to EOF1 because of its poleward propagation and can eventually project negatively onto it. Thus, the low or negative values of the cross covariance between $m$ and PC1 in the strong lower-level baroclinicity case correspond to a response of the eddies that does not maintain the jet shift associated with EOF1 but rather makes the jet propagate poleward.

The poleward-propagation regime was found to emerge and dominate in the simulations of Son and Lee (2006), 

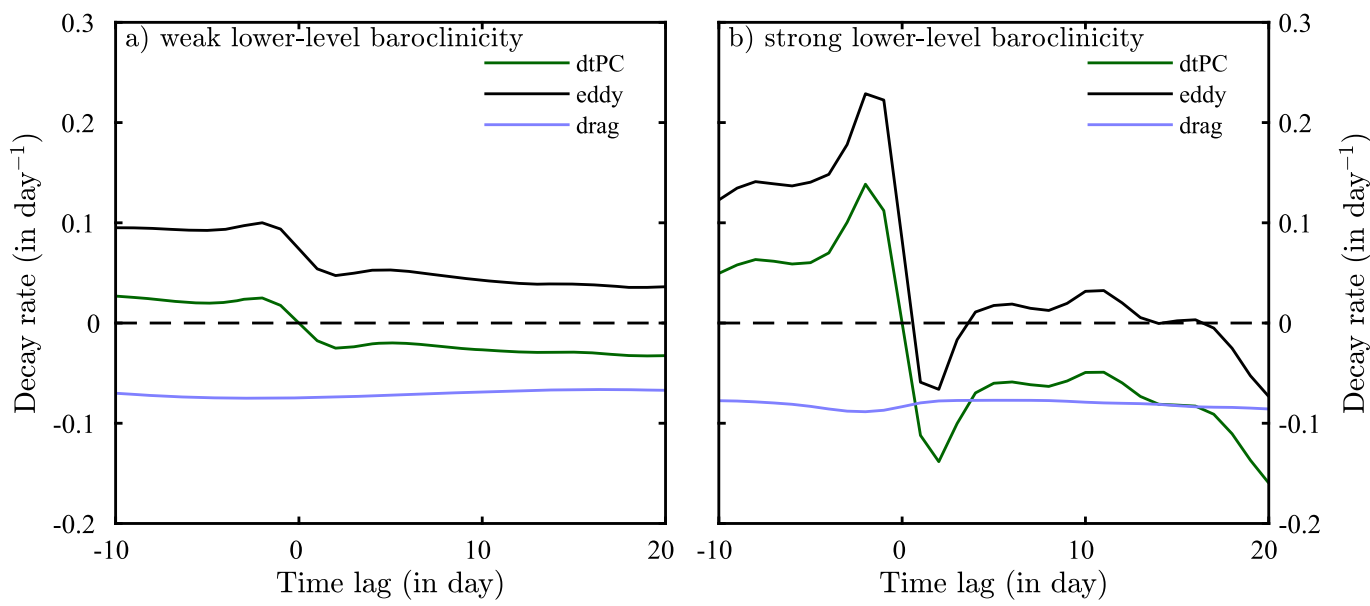

FIG. 4. Rate of change of the PC1 autocorrelation function [left-hand side of Eq. (5); green], and its momentum $\left[\tau_{m}^{-1}\right.$ of Eq. (5); black] and drag contribution [ $\tau_{d}^{-1}$ of Eq. (5); blue], for two simulations of LB25: (a) weak baroclinicity $\left(U_{2}-U_{3}=0.2 U_{0}\right)$ and (b) strong baroclinicity $\left(U_{2}-U_{3}=0.4 U_{0}\right)$.

Lee et al. (2007), and Son et al. (2008) forced by a strong high-latitude cooling rate, that is, when the midlatitude baroclinic zone was reinforced and enlarged. This is consistent with our simulations, which show the dominance of the poleward-propagation regime when the midlatitude lower-level baroclinicity is increased. The rationale for the poleward-propagation regime provided by Lee et al. (2007) is the following:

- A wave generated in the jet core, which propagates equatorward, induces momentum convergence near the jet core and a divergence on its equatorward flank.

- The momentum flux divergence decelerates the zonal wind near the critical latitude, which moves it poleward.

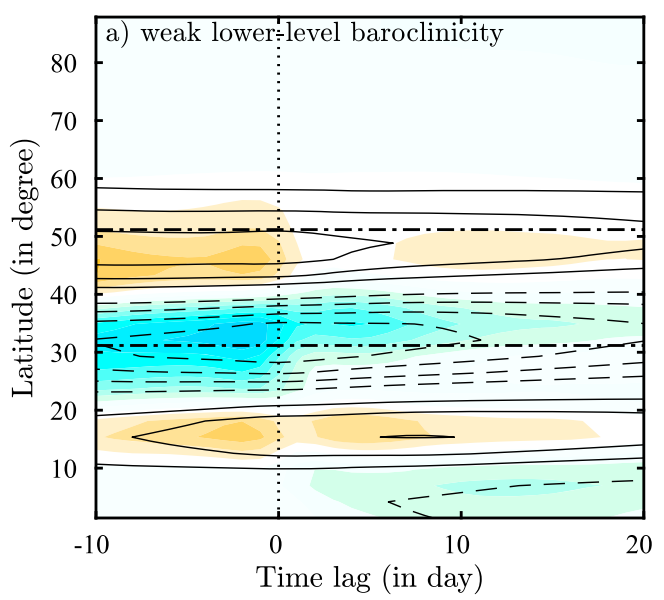

- The next equatorward-propagating wave will therefore break slightly farther away from the equator; its momentum convergence and divergence patterns are shifted poleward compared to the first wave. Thereby, the jet is farther pushed poleward and so on.

This mechanism needs the Rossby waves to reach a critical latitude on the equatorward flank of the jet and to strongly decelerate the westerlies there. This is unlikely to happen when waves do not propagate away from the jet and the jet somehow acts as a waveguide. This assertion can be tested by comparing the structure of Rossby waves between a weak and strong lowerlevel baroclinicity simulation. Figure 6 shows one-point correlation maps of the meridional wind for both

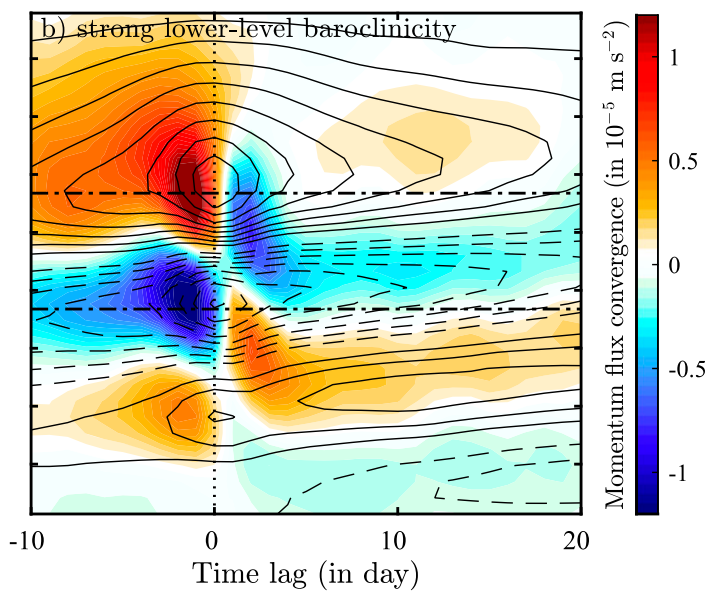

FIG. 5. Lagged regression onto PC1 of anomalous zonally and vertically averaged eddy momentum flux convergence (shaded) and zonal wind (contours; interval: $0.5 \mathrm{~m} \mathrm{~s}^{-1}$ ) as a function of lag and latitude for two simulations of LB25: (a) weak baroclinicity $\left(U_{2}-U_{3}=0.2 U_{0}\right)$ and (b) strong baroclinicity $\left(U_{2}-U_{3}=0.4 U_{0}\right)$. The black dashed-dotted lines indicate the meridional extension of the mean jet (taken as $\pm 10^{\circ}$ around jet maximum). 

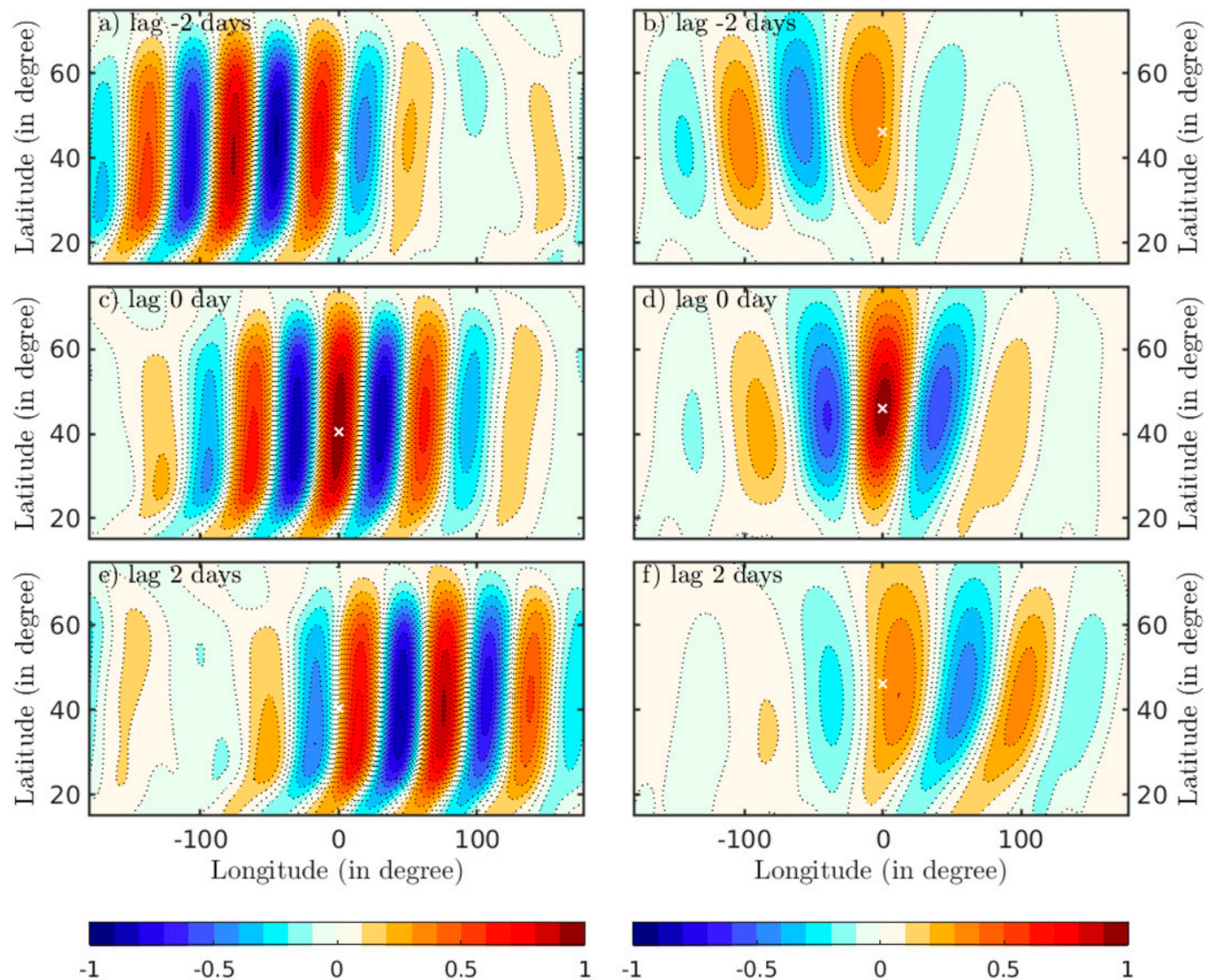

0

0.5

1

FIG. 6. Lagged one-point correlation map of the meridional wind for (a),(c),(e) weak lower-level baroclinicity $\left(U_{2}-U_{3}=0.2 U_{0}\right)$ and (b),(d),(f) strong lower-level baroclinicity $\left(U_{2}-U_{3}=0.4 U_{0}\right)$. These correlations maps have been plotted with lags of (a),(b) -2 , (c),(d) 0 , and (e),(f) +2 days. The reference point (white cross) is, in each simulation, close to the maximum of zonal wind.

simulations with different lags: $-2,0$, and +2 days. On the one hand, the weak lower-level baroclinicity simulation (Figs. 6a,c,e) shows a prevalence of zonal wavenumber $k=6$. The waves are slightly anticyclonically tilted, as expected from spherical geometry and the quasigeostrophic model bias (Rivière 2009). Therefore, Rossby wave propagation is almost zonal, waves staying confined into the jet (cf. Figs. 6a and 6e), which mainly acts as a waveguide. On the other hand, the strong lower-level baroclinicity simulation (Figs. 6b,d,f) is dominated by wavenumber $k=4$, and the observed wave structure has more pronounced tilts: a cyclonic tilt for the negative lag (Fig. 6b) and an even more pronounced anticyclonic tilt at the positive lag (Fig. 6f). At lag 0 days (Fig. 6d), waves seem to exhibit both tilts: cyclonic west from the reference point and anticyclonic east of it. This could indicate wave reflections occurring on the northern flank of the jet, which is consistent with the change in sign of the momentum flux convergence at short positive lags in Fig. 5b (Rivière et al. 2016).
In any case, Fig. 6 reveals that waves tend to propagate more meridionally for the strong lower-level simulation. The stronger anticyclonic tilt observed there induces zonal wind deceleration on the equatorward side of the jet as needed in the above mechanism of poleward propagation.

This difference in wave structures can be related to the shape of the jet. Meridionally propagating waves are more likely to occur within broader jets for which the PV gradient is smoother and does not decrease too fast on the flanks of the jet while meridionally trapped waves are confined in well-defined regions of strong PV gradient (Martius et al. 2010). This picture is confirmed by Fig. 7, which shows the time-mean zonal wind and PV gradient for LB25. The width of the jet increases while its peak amplitude stays roughly constant when the baroclinicity increases (Fig. 7a). For all simulations, the PV gradient has two peaks (Fig. 7b), a strong one located near the jet core and a weaker one closer to the equator. The two peaks are stronger when the 

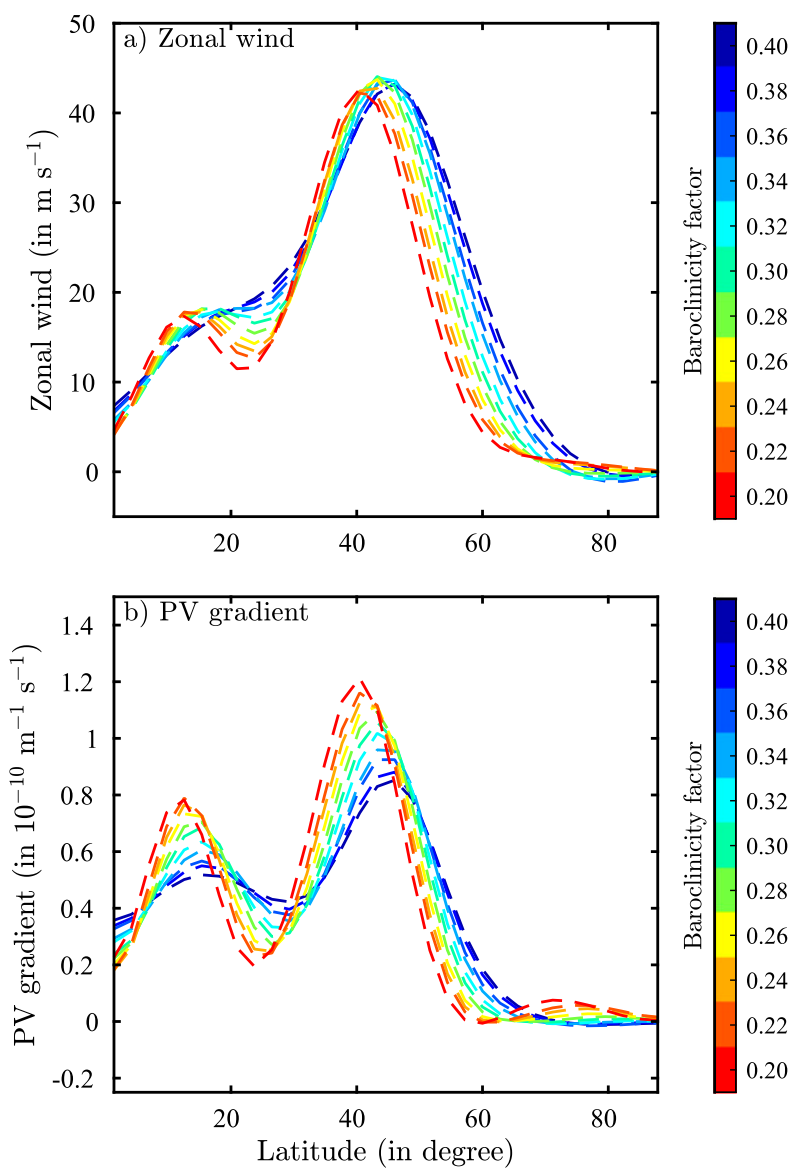

FIG. 7. Climatological mean of (a) the zonally averaged zonal wind and (b) zonally averaged PV gradient at $200 \mathrm{hPa}$ for LB25, the baroclinicity factor being $\left(U_{2}-U_{3}\right) / U_{0}$.

lower-level baroclinicity decreases, and they also seem to be sharper. To confirm the latter statement, an estimation of the width at half maximum was computed for each simulation (Fig. 8c). It clearly shows a broader PV gradient as the lower-level baroclinicity increases. If the same computation is made for the UB25 simulations (Fig. 8a), the PV gradient is found to have a constant width, which supports our interpretation and the fact that changes in upper-level baroclinicity does not influence the poleward propagation.

However, one may argue that the broader climatological mean jet and PV gradient is due to smearing by the larger meridional incursions when poleward propagation of zonal wind anomalies occur. It is thus not clear if a wider climatological mean jet necessarily means a wider jet at different times of the simulation. To address this issue, the width of the zonally averaged PV gradient has been computed at each day of the simulations and then averaged. The result is shown in Fig. 8d (and Fig. 8b for UB25), which still clearly shows that the averaged width at half maximum also increases with the lower-level baroclinicity and not with the upperlevel baroclinicity. Therefore, a stronger lower-level baroclinicity leads to a broader jet and a smoother PV gradient, enabling the poleward-propagation regime to become the dominant mode of variability. It confirms the interpretation provided by Lee et al. (2007) and Son et al. (2008). In their case, the poleward-propagation regime associated with the broader and weaker PV gradient logically emerges when the baroclinic zone is forced to be broader by a stronger cooling rate at high latitudes. In our case, it is not straightforward to explain why the PV gradient and the jet broaden when the restoration baroclinicity is intensified and the meridional width of the restoration baroclinicity is kept unchanged.

Two possible mechanisms explaining how a stronger low-level (but not upper level) baroclinicity leads to a broader jet can be put forward. The first one relies on baroclinic instability arguments. The development of baroclinic instability requires a change of sign of the PV gradient in the vertical; given that this gradient is always positive at upper levels, this is equivalent to having a negative PV gradient in the lower troposphere. An increase in lower-level baroclinicity will tend to enhance the negative low-level PV gradient and therefore widen the latitudinal band in which it is negative, as shown in Figs. $9 \mathrm{c}$ and $9 \mathrm{~d}$ for different restoration profiles [see Eq. (2) for their definition]. In contrast, a change in upper-level baroclinicity does not affect the width of the region where the $\mathrm{PV}$ gradient changes sign in the vertical, as it remains positive at upper levels (Figs. 9a and $9 \mathrm{~b}$ ). So the latitudinal band in which baroclinic waves might be unstable increases in width only when the lower-level baroclinicity increases. This will in turn increase the width of the latitudinal band of eddy momentum deposition in the upper troposphere and thus the width of the eddy-driven jet.

The second potential explanation relies on changes in eddy wavelengths. There is a clear shift toward larger eddy length scales for stronger lower-level baroclinicity (cf. Figs. 6b,d,f and Figs. 6a,c,e). Figure 10 confirms it by showing the spectrum of the squared meridional wind as a function of latitude and wavenumber for a weak and a strong lower-level baroclinicity simulation. This figure also confirms the assumption that waves are trapped in the jet for a weak lower-level baroclinicity (Fig. 10a), whereas they can escape and propagate meridionally for a stronger baroclinicity (Fig. 10c). Moreover, waves tend to reach higher amplitudes for a stronger lower-level baroclinicity and at all latitudes (cf. Figs. 10b and 10d). The increase in eddy horizontal scale when the baroclinicity increases is consistent with similar quasigeostrophic or primitive equation simulations 

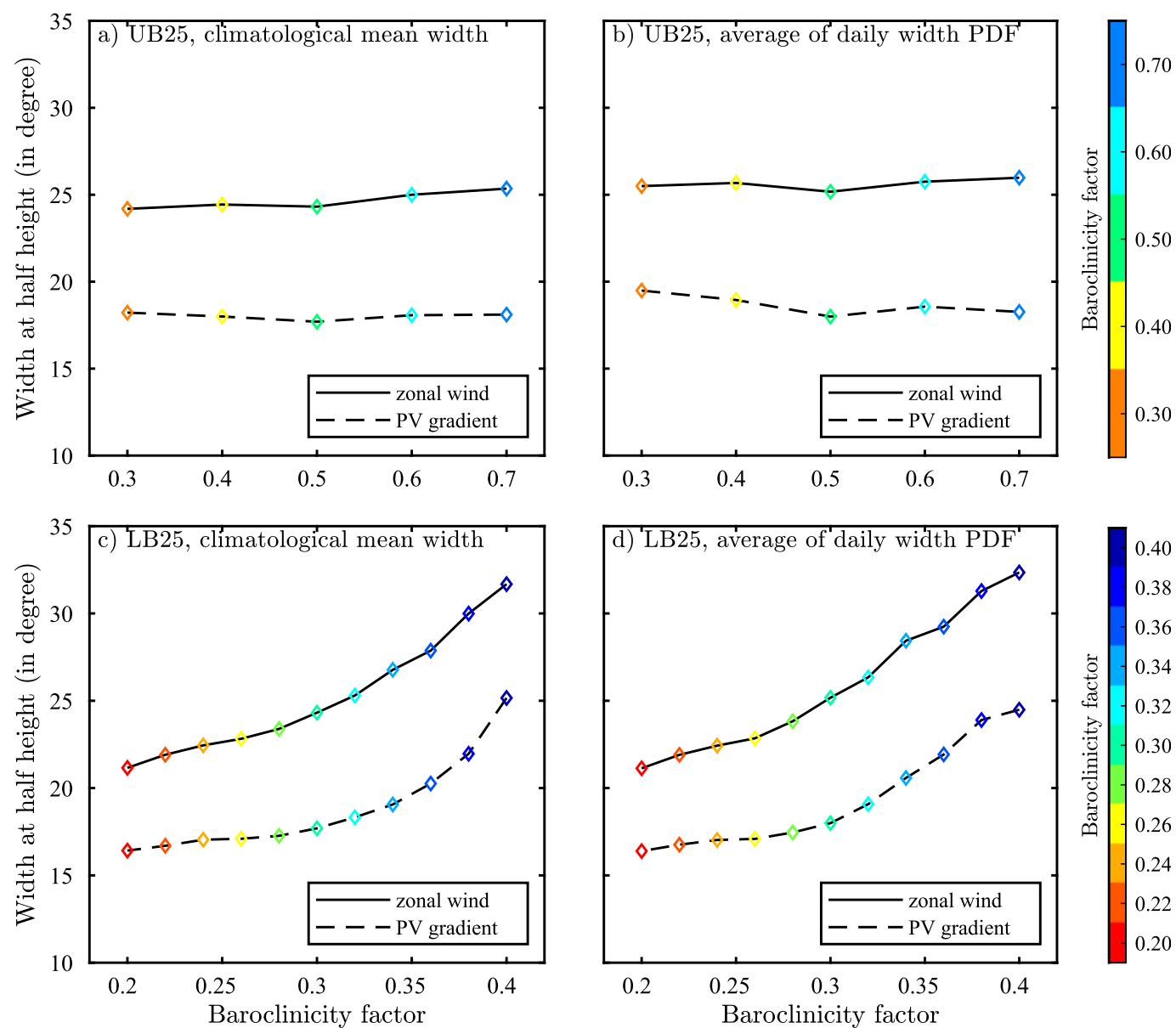

FIG. 8. (a),(c) Full width at half maximum of the main peak of zonally averaged zonal wind (solid line) and PV gradient (dashed line) at $200 \mathrm{hPa}$ and (b),(d) averaged full width at half maximum of the daily peak of the same quantities as a function of (a),(b) the upper-level baroclinicity factor $\left[\left(U_{1}-U_{2}\right) / U_{0}\right]$ for each simulation of UB25 and (c),(d) the lower-level baroclinicity factor $\left[\left(U_{2}-U_{3}\right) / U_{0}\right]$ for each simulation of LB25 (plotted with their respective color).

(Whitaker and Barcilon 1995; Chai and Vallis 2014). However, there are different competing mechanisms to explain this result, and the mechanism at play may depend on the range of the baroclinicity (or criticality) values. One possible explanation might be the upscale nonlinear energy transfer. Indeed, an increase in the lower-level baroclinicity enhances wave generation of the most unstable synoptic waves. This intensifies nonlinear interaction among synoptic waves that lead to more energy transfer toward large waves. There are also linear and alternative nonlinear arguments that may explain this result, which are particularly more relevant when discussing weakly nonlinear regimes, as discussed in the two previously cited studies. So one possible interpretation for the broader jet in the presence of the stronger lower-level baroclinicity is the following: by increasing the baroclinicity, waves increase in scale, so they deposit momentum over a broader region. This broadens the jet, which in turn favors more the poleward-propagation regime. We have checked that the upper-level baroclinicity has a less drastic effect on eddy length scales in the present simulations (not shown).

Finally, this shift toward smaller wavenumbers for strong lower-level baroclinicity could explain another result: the stronger negative values of eddy momentum forcing observed at short positive lags (Fig. 3b). Smaller wavenumbers means more planetary waves, which tend to reflect near the jet flank and induce a negative eddy momentum forcing (Rivière et al. 2016; RRC17). It could seem paradoxical that more reflections occur for strong lower-level baroclinicity when the jet acts less as a waveguide. However, in that case, equatorwardpropagating planetary waves may reflect or be absorbed depending on the situation. The pronounced tilt observed on Fig. 6f (and to a lesser extent on Fig. 6d) is 

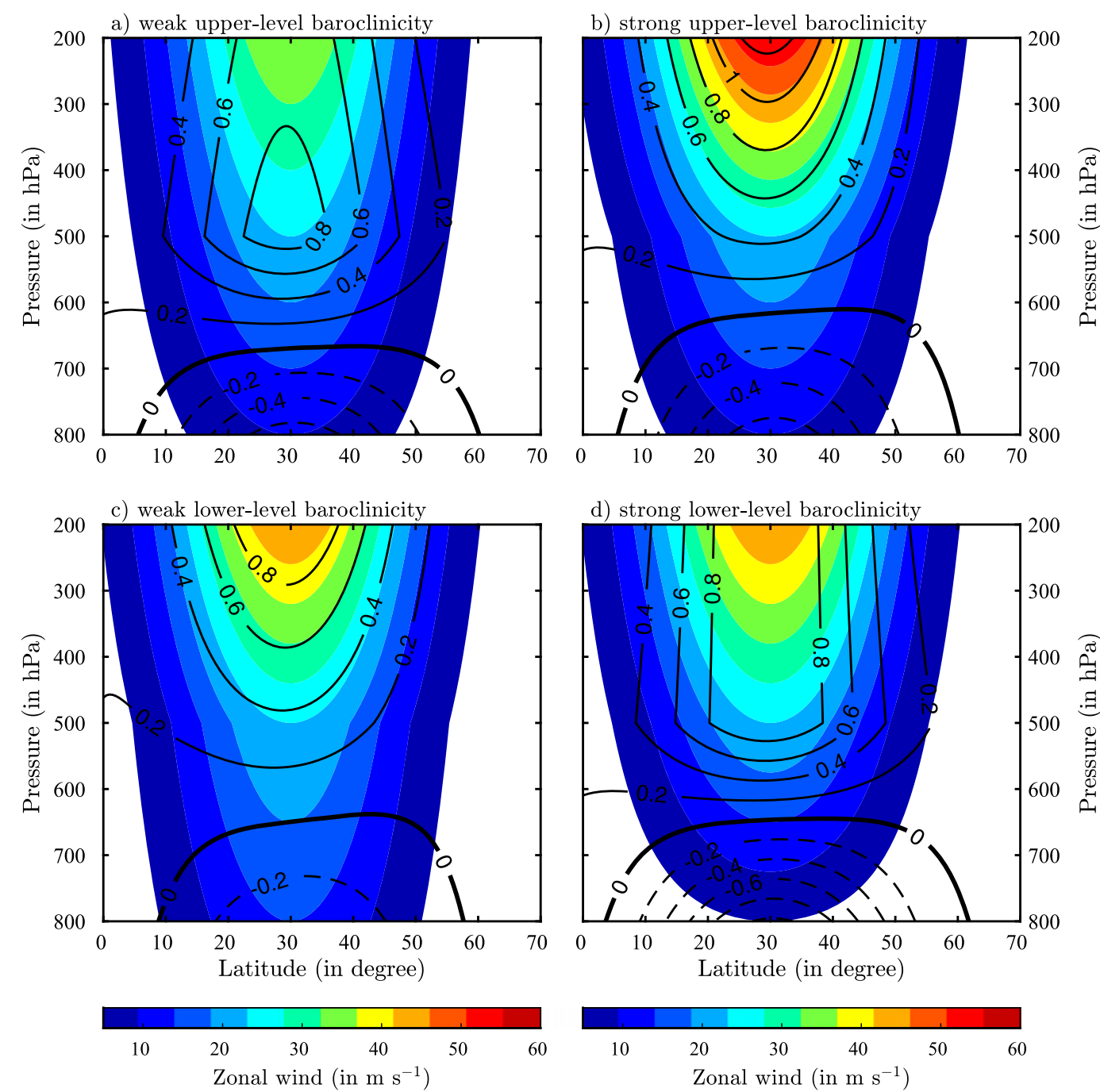

FIG. 9. Vertical cross section of the zonal wind (shaded) and PV gradient (contours; interval: $2 \times 10^{-11} \mathrm{~m}^{-1} \mathrm{~s}^{-1}$ ) of the restoration basic states for (a) weak upper-level baroclinicity $\left(U_{1}-U_{2}=0.4 U_{0}\right)$, (b) strong upper-level baroclinicity $\left(U_{1}-U_{2}=0.7 U_{0}\right)$, (c) weak lower-level baroclinicity $\left(U_{2}-U_{3}=0.2 U_{0}\right)$, and (d) strong lower-level baroclinicity $\left(U_{2}-U_{3}=0.4 U_{0}\right)$.

consistent with an increase in the wave-mean flow interaction described in Rivière et al. (2016), leading sometimes to the appearance of reflecting levels before the wave reaches the critical latitude. This well-marked equatorward wave propagation, which may result in reflection or absorption, does not appear for weak lower-level baroclinicity where waves are more systematically trapped in the jet core by the sharp PV gradient (Figs. 6c and 6e).

\section{d. Additional sensitivity experiments}

\section{1) BAROCLINICITY INTENSITY VERSUS LATITUDE}

As recalled in the introduction, changes in the persistence of the leading mode of jet variability have been linked to changes in the mean jet latitude (e.g., Kidston and Gerber 2010; Arakelian and Codron 2012), and one may argue that the results from the LB25 experiments could also be due to changes in the mean jet latitude. To check this, the LB25 series is compared to JP25, a series of simulations where the intensity of the restoration baroclinicity is fixed but its latitude is varied. To compare these two series, we compute the instantaneous rate of change of the autocorrelation function due to the eddy momentum forcing [term $\tau_{m}^{-1}$ from Eq. (5)]. Its averaged value between lag +5 and +15 days is plotted on Fig. 11 for all simulations. We first recover in the JP25 experiments the classical result (Barnes et al. 2010; Arakelian and Codron 2012) that, as the latitude of the mean jet increases, the eddy feedback 

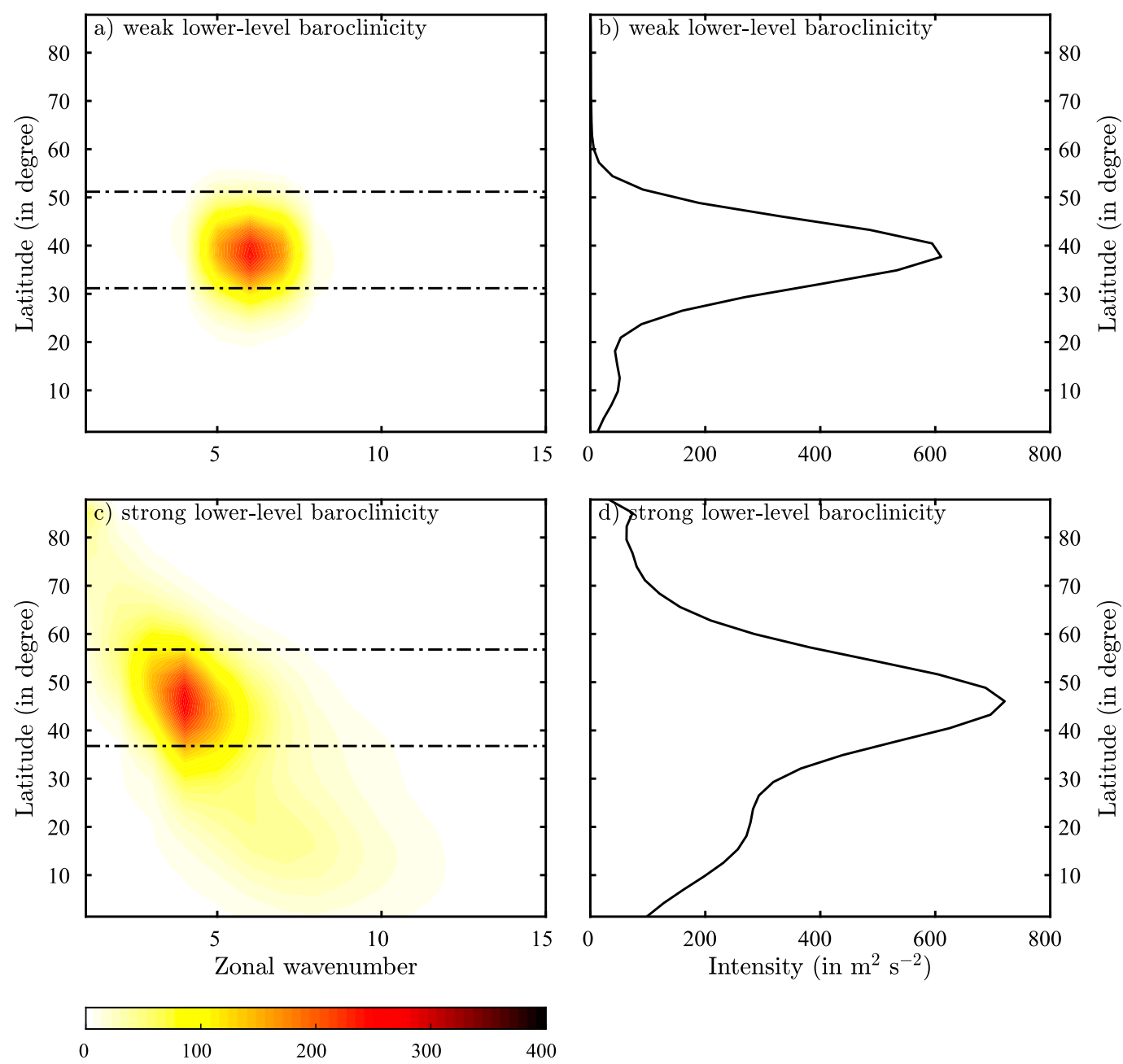

FIG. 10. (a),(c) Spectrum of the squared meridional wind as a function of latitude and zonal wavenumber and (b),(d) its integral over wavenumber for (a),(b) a weak lower-level baroclinicity $\left(U_{2}-U_{3}=0.2 U_{0}\right)$ and (c),(d) a strong lower-level baroclinicity $\left(U_{2}-U_{3}=0.4 U_{0}\right)$.

coefficient decreases (black squares). The LB25 experiments indicate that, as the lower-level baroclinicity increases, the jet moves slightly poleward, consistent with Butler et al. (2010) and Rivière (2011). For these experiments, the eddy feedback also decreases as the jet moves poleward but with a much steeper slope than for the JP25 experiments. This strongly suggests that the impact of the lower-level baroclinicity onto the EOF1 persistence cannot be simply explained by the latitudinal displacement of the jet.

\section{2) SENSitivity to RELAXATION TIME SCALES}

To check the robustness of the results, the same series of simulations as LB25 and UB25 has been run but with the relaxation time scales set to the same values as in RRC17: $\tau_{R_{1}}=40$ days and $\tau_{R_{2}}=15$ days. These setups, denoted as LB40 and UB40 (cf. Table 1), are also more similar to the Held and Suarez's (1994) benchmark. Figure 12 shows the PC1 autocorrelation for these new series of simulations. As for LB25, the persistence of the leading EOF generally increases by decreasing the lower-level baroclinicity while, as for UB25, this persistence is not impacted by a change in upper-level baroclinicity. There is only one exception for the very weak baroclinicity $\left[\left(U_{2}-U_{3}\right) / U_{0}\right.$ equal to 0.20 and 0.22$]$ for which the persistence suddenly decreases from 0.22 to 0.20 . The general increased persistence as the baroclinicity decreases can be explained by the increased eddy momentum forcing $\tau_{m}^{-1}$ (Fig. 13b) as for the LB25 experiments. Only the marginal cases for lower-level baroclinicity factors from 0.22 to 0.20 show an abrupt reversal of the tendency. Similar to UB25, the eddy momentum forcing does not show significant change in average when the upper-level baroclinicity is increased 


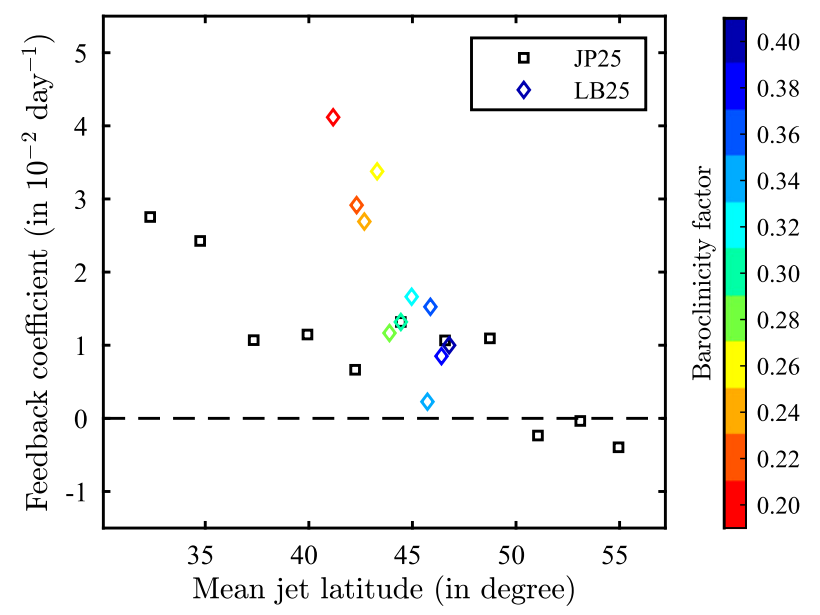

FIG. 11. Feedback coefficient as a function of mean jet position for the JP25 series (black squares) and the LB25 series (colored diamonds), the baroclinicity factor being $\left(U_{2}-U_{3}\right) / U_{0}$.

(Fig. 13a). In conclusion, the results found previously for $\tau_{R_{1}}=25$ days and $\tau_{R_{2}}=25$ days are still valid here, except for the very weak lower-level baroclinicity simulations.

\section{Conclusions}

The impact of changes in upper- and lower-level baroclinicity on the eddy-driven jet variability has been investigated using a three-level quasigeostrophic model on the sphere. This study focused on the persistence of the leading EOF of the zonally and vertically averaged zonal wind. We found that its sensitivity to the upper or lower baroclinicity is very different: an increase in the upper-level baroclinicity has no significant effect whereas an increase in lower-level baroclinicity tends to decrease the EOF1 persistence.

When the lower-level baroclinicity increases, EOF1 also becomes more characterized by a poleward propagation of zonal wind anomalies. Using the terminology introduced by Son and Lee (2006), the zonal index regime is progressively replaced by the polewardpropagation regime. In the zonal index regime, the meridional excursion of the jet is maintained by a strong positive eddy feedback. In the poleward-propagation regime, the eddies instead deposit their momentum poleward of the current jet position, leading to its propagation. The interpretation is provided in terms of PV gradient. For strong lower-level baroclinicity, the PV gradient is broad and weak enough to favor an equatorward propagation of the waves. Then the waves decelerate the zonal winds on the equatorward flank of the jet when they break and displace the critical latitude further poleward. This tends to shift the eddy
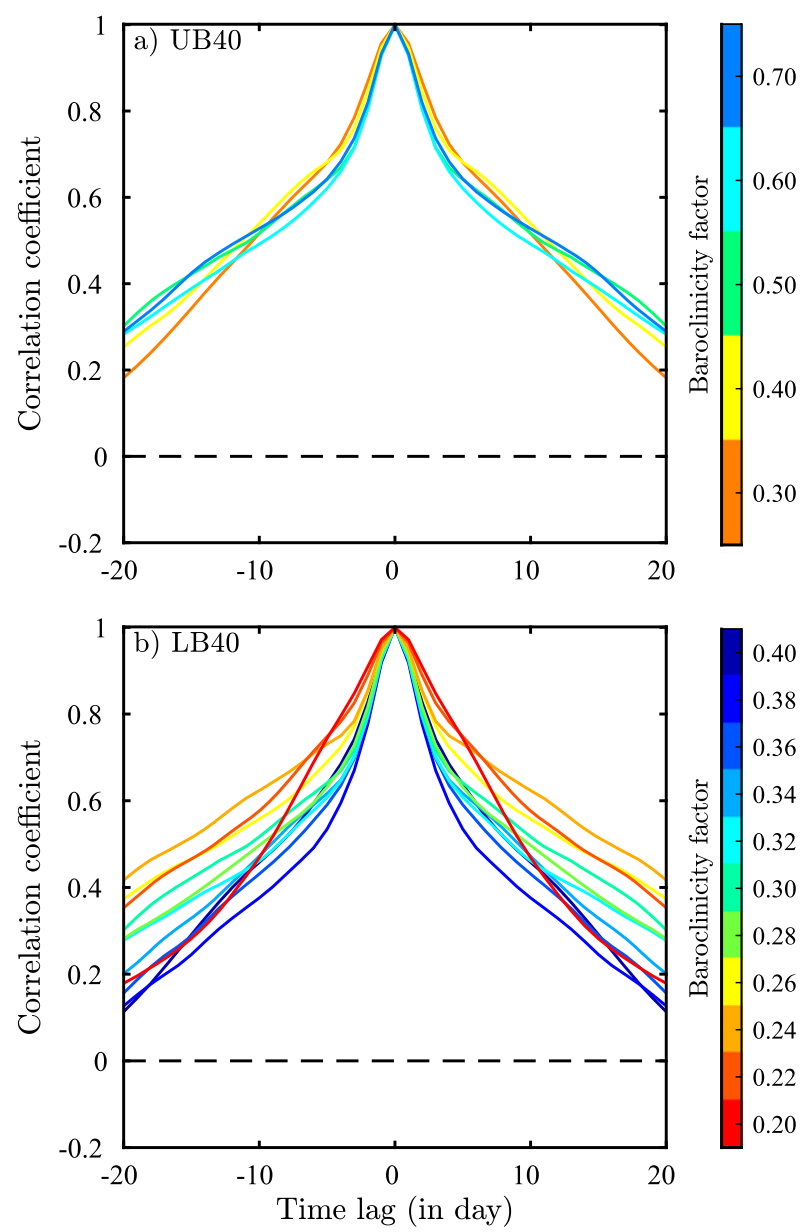

FIG. 12. As in Fig. 2, but for UB40 and LB40 series.

momentum flux convergence-divergence patterns and the zonal wind anomalies poleward with time. For weak lower-level baroclinicity, the PV gradient is so strong and sharp that the jet acts as a waveguide, waves are trapped, and the deceleration of the zonal winds on the equatorward flank of the jet is too weak to modify the location of the critical latitudes. The interpretation is similar to Lee et al. (2007) and Son et al. (2008), but the setup of the numerical experiments is different. In the previous studies, a broader PV gradient logically appears as the width of the forced baroclinic zone increases. In our case, an intensification of the restoration baroclinicity without changing its meridional width is enough to lead to the same effect. Two explanations are provided. The first one is based on a baroclinic instability argument. When the lower-level baroclinicity increases, the latitudinal band where baroclinic instability is likely to occur increases, which enlarges the region of momentum deposit by the waves and hence widens the jet. The second one is based on the increased wavelength when the lower-level baroclinicity increases. 

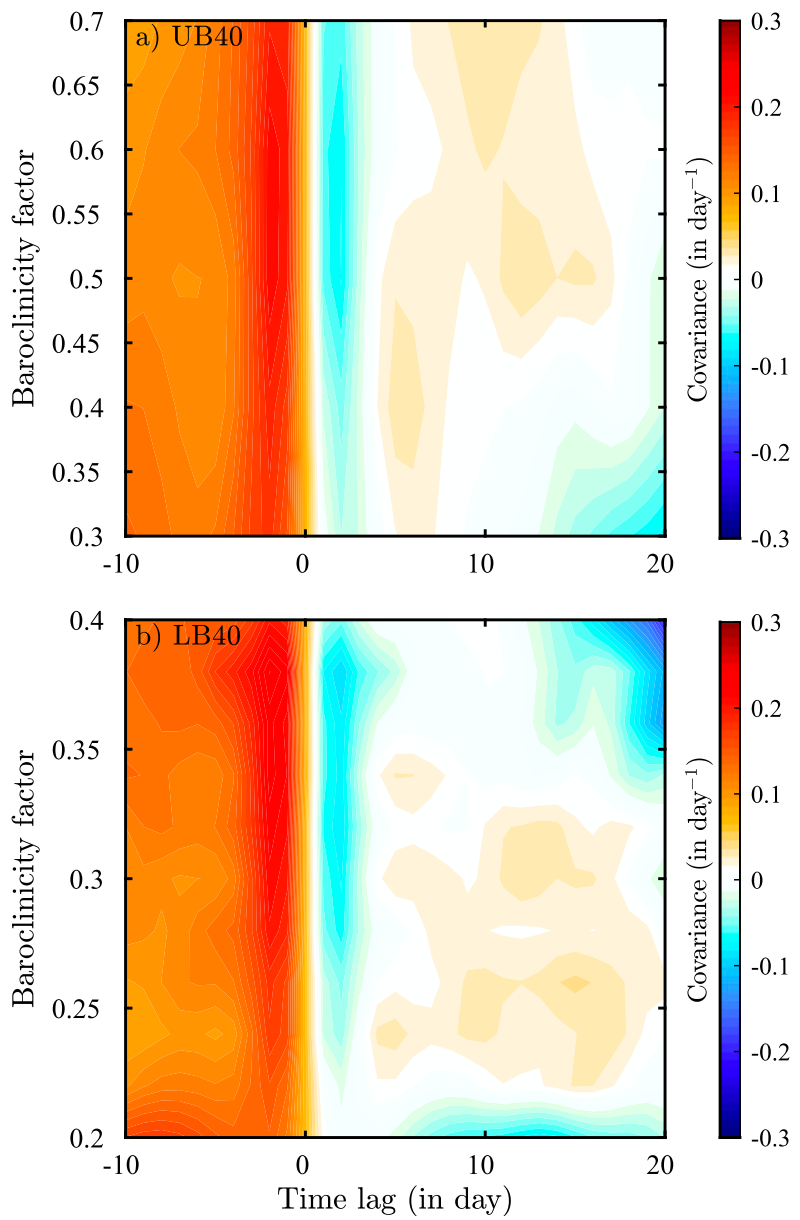

FIG. 13. As in Fig. 3, but for UB40 and LB40 series.

As larger waves deposit momentum over a larger latitudinal band, the eddy-driven jet becomes broader.

Another effect of the increased wavelength when the lower-level baroclinicity increases is seen on shorter time scales. In the strong lower-level baroclinicity cases, the wave scale becomes larger, and waves are more inclined to be reflected onto the equatorward side of the jet, reinforcing the negative eddy feedback a few days after the peak of PC1 (Rivière et al. 2016; RRC17). This constitutes an additional reason for the weaker persistence of $\mathrm{PC} 1$ for stronger lower-level baroclinicity.

The present idealized study provides dynamical diagnoses to further investigate the persistence of annular modes in future climate projections obtained with the Coupled Model Intercomparison Project (CMIP) exercises. In addition to the sensitivity to the jet latitude already discussed in several papers (Kidston and Gerber 2010; Arakelian and Codron 2012; Barnes and Polvani 2013), the sensitivity to the lower-level baroclinicity and to the width of the PV gradient should be examined, especially during autumn and early winter when the polar amplification is expected to be the strongest in the Northern Hemisphere.

Acknowledgments. The authors thank Jian Lu and two anonymous reviewers for their relevant and helpful remarks. This work was partially funded by project MORDICUS (ANR-13-SENV-0002) from the French National Research Agency.

\section{REFERENCES}

Arakelian, A., and F. Codron, 2012: Southern Hemisphere jet variability in the IPSL GCM at varying resolutions. J. Atmos. Sci., 69, 3788-3799, https://doi.org/10.1175/JAS-D-12-0119.1.

Barnes, E. A., and D. L. Hartmann, 2011: Rossby wave scales, propagation, and the variability of eddy-driven jets. J. Atmos. Sci., 68, 2893-2908, https://doi.org/10.1175/JAS-D-11-039.1.

- and L. M. Polvani, 2013: Response of the midlatitude jets, and of their variability, to increased greenhouse gases in the CMIP5 models. J. Climate, 26, 7117-7135, https://doi.org/10.1175/ JCLI-D-12-00536.1.

- D. L. Hartmann, D. M. W. Frierson, and J. Kidston, 2010: Effect of latitude on the persistence of eddy-driven jets. Geophys. Res. Lett., 37, L11804, https://doi.org/10.1029/ 2010GL043199.

Butler, A. H., D. W. J. Thompson, and R. Heikes, 2010: The steadystate atmospheric circulation response to climate change-like thermal forcings in a simple general circulation model. $\mathrm{J}$. Climate, 23, 3474-3496, https://doi.org/10.1175/2010JCLI3228.1.

Chai, J., and G. K. Vallis, 2014: The role of criticality on the horizontal and vertical scales of extratropical eddies in a dry GCM. J. Atmos. Sci., 71, 2300-2318, https://doi.org/10.1175/JAS-D-13-0351.1.

Chavaillaz, Y., F. Codron, and M. Kageyama, 2013: Southern westerlies in LGM and future (RCP4.5) climates. Climate Past, 9, 517-524, https://doi.org/10.5194/cp-9-517-2013.

Harvey, B. J., L. C. Shaffrey, and T. J. Woollings, 2014: Equator-topole temperature differences and the extra-tropical storm track responses of the CMIP5 climate models. Climate Dyn., 43, 1171-1182, https://doi.org/10.1007/s00382-013-1883-9.

Held, I. M., and E. O'Brien, 1992: Quasigeostrophic turbulence in a three-layer model: Effects of vertical structure in the mean shear. J. Atmos. Sci., 49, 1861-1870, https://doi.org/10.1175/ 1520-0469(1992)049<1861:QTIATL > 2.0.CO;2.

_ , and M. J. Suarez, 1994: A proposal for the intercomparison of the dynamical cores of atmospheric general circulation models. Bull. Amer. Meteor. Soc., 75, 1825-1830, https://doi.org/10.1175/ 1520-0477(1994)075<1825:APFTIO > 2.0.CO;2.

Hoskins, B. J., and P. J. Valdes, 1990: On the existence of stormtracks. J. Atmos. Sci., 47, 1854-1864, https://doi.org/10.1175/ 1520-0469(1990)047<1854:OTEOST>2.0.CO;2.

Kidston, J., and E. P. Gerber, 2010: Intermodel variability of the poleward shift of the austral jet stream in the CMIP3 integrations linked to biases in 20th century climatology. Geophys. Res. Lett., 37, L09708, https://doi.org/10.1029/2010GL042873.

Lee, S., S.-W. Son, K. Grise, and S. B. Feldstein, 2007: A mechanism for the poleward propagation of zonal mean flow anomalies. J. Atmos. Sci., 64, 849-868, https://doi.org/10.1175/JAS3861.1.

Lindzen, R. S., and B. Farrell, 1980: A simple approximate result for the maximum growth rate of baroclinic instabilities. $J$. Atmos. Sci., 37, 1648-1654, https://doi.org/10.1175/1520-0469(1980) $037<1648$ :ASARFT $>2.0 . \mathrm{CO} ; 2$. 
Lorenz, D. J., and D. L. Hartmann, 2001: Eddy-zonal flow feedback in the Southern Hemisphere. J. Atmos. Sci., 58, 33123327, https://doi.org/10.1175/1520-0469(2001)058<3312:EZFFIT > 2.0.CO;2.

— Hemisphere winter. J. Climate, 16, 1212-1227, https://doi.org/ 10.1175/1520-0442(2003)16<1212:EFFITN>2.0.CO;2.

Lu, J., G. Chen, and D. M. W. Frierson, 2008: Response of the zonal mean atmospheric circulation to El Niño versus global warming. J. Climate, 21, 5835-5851, https://doi.org/10.1175/ 2008JCLI2200.1.

Marshall, J., and F. Molteni, 1993: Toward a dynamical understanding of planetary-scale flow regimes. J. Atmos. Sci., $\mathbf{5 0}$, 1792-1818, https://doi.org/10.1175/1520-0469(1993)050<1792: TADUOP $>2.0 . \mathrm{CO} ; 2$.

Martius, O., C. Schwierz, and H. C. Davies, 2010: Tropopause-level waveguides. J. Atmos. Sci., 67, 866-879, https://doi.org/10.1175/ 2009JAS2995.1.

Michel, C., and G. Rivière, 2014: Sensitivity of the position and variability of the eddy-driven jet to different SST profiles in an aquaplanet general circulation model. J. Atmos. Sci., 71, 349371, https://doi.org/10.1175/JAS-D-13-074.1.

Ogawa, F., H. Nakamura, K. Nishii, T. Miyasaka, and A. KuwanoYoshida, 2016: Importance of midlatitude oceanic frontal zones for the annular mode variability: Interbasin differences in the southern annular mode signature. J. Climate, 29, 61796199, https://doi.org/10.1175/JCLI-D-15-0885.1.

Oudar, T., E. Sanchez-Gomez, F. Chauvin, J. Cattiaux, L. Terray, and C. Cassou, 2017: Respective roles of direct GHG radiative forcing and induced Arctic sea ice loss on the Northern Hemisphere atmospheric circulation. Climate Dyn., 49, 3693 3713, https://doi.org/10.1007/s00382-017-3541-0.

Rivière, G., 2009: The effect of latitudinal variations in low-level baroclinicity on eddy life cycles and upper-tropospheric wave-breaking processes. J. Atmos. Sci., 66, 1569-1592, https://doi.org/10.1175/2008JAS2919.1.

_ 2011: A dynamical interpretation of the poleward shift of the jet streams in global warming scenarios. J. Atmos. Sci., 68 , 1253-1272, https://doi.org/10.1175/2011JAS3641.1.

_ L L. Robert, and F. Codron, 2016: A short-term negative eddy feedback on midlatitude jet variability due to planetary waves reflections. J. Atmos. Sci., 73, 4311-4328, https://doi.org/ 10.1175/JAS-D-16-0079.1.

Robert, L., G. Rivière, and F. Codron, 2017: Positive and negative eddy feedbacks acting on midlatitude jet variability in a threelevel quasigeostrophic model. J. Atmos. Sci., 74, 1635-1649, https://doi.org/10.1175/JAS-D-16-0217.1.
Seager, R., N. Harnik, Y. Kushnir, W. A. Robinson, and J. A. Miller, 2003: Mechanisms of hemispherically symmetric climate variability. J. Climate, 16, 2960-2978, https://doi.org/10.1175/ 1520-0442(2003)016<2960:MOHSCV >2.0.CO;2.

Shaw, T. A., and Coauthors, 2016: Storm track processes and the opposing influences of climate change. Nat. Geosci., 9, 656664, https://doi.org/10.1038/ngeo2783.

Simpson, I. R., and L. M. Polvani, 2016: Revisiting the relationship between jet position, forced response, and annular mode variability in the southern midlatitudes. Geophys. Res. Lett., 43, 2896-2903, https://doi.org/10.1002/2016GL067989.

—, P. Hitchcock, T. G. Shepherd, and J. F. Scinocca, 2013a: Southern annular mode dynamics in observations and models. Part I: The influence of climatological zonal wind biases in a comprehensive GCM. J. Climate, 26, 3953-3967, https://doi.org/ 10.1175/JCLI-D-12-00348.1.

, T. G. Shepherd, P. Hitchcock, and J. F. Scinocca, 2013b: Southern annular mode dynamics in observations and models. Part II: Eddy feedbacks. J. Climate, 26, 5220-5241, https://doi.org/10.1175/JCLI-D-12-00495.1.

_ T. T. Ahaw, and R. Seager, 2014: A diagnosis of the seasonally and longitudinally varying midlatitude circulation response to global warming. J. Atmos. Sci., 71, 2489-2515, https:// doi.org/10.1175/JAS-D-13-0325.1.

Son, S.-W., and S. Lee, 2005: The response of westerly jets to thermal driving in a primitive equation model. J. Atmos. Sci., 62, 3741-3757, https://doi.org/10.1175/JAS3571.1.

—, and —, 2006: Preferred modes of variability and their relationship with climate change. J. Climate, 19, 2063-2075, https://doi.org/10.1175/JCLI3705.1.

,,-- S. B. Feldstein, and J. E. T. Hoeve, 2008: Time scale and feedback of zonal-mean-flow variability. J. Atmos. Sci., 65 , 935-952, https://doi.org/10.1175/2007JAS2380.1.

Vallis, G. K., P. Zurita-Gotor, C. Cairns, and J. Kidston, 2015: Response of the large-scale structure of the atmosphere to global warming. Quart. J. Roy. Meteor. Soc., 141, 1479-1501, https://doi.org/10.1002/qj.2456.

Whitaker, J. S., and A. Barcilon, 1995: Low-frequency variability and wavenumber selection in models with zonally symmetric forcing. J. Atmos. Sci., 52, 491-503, https://doi.org/10.1175/ 1520-0469(1995)052<0491:LFVAWS >2.0.CO;2.

Yuval, J., and Y. Kaspi, 2016: Eddy activity sensitivity to changes in the vertical structure of baroclinicity. J. Atmos. Sci., 73, 17091726, https://doi.org/10.1175/JAS-D-15-0128.1.

Zurita-Gotor, P., 2014: On the sensitivity of zonal-index persistence to friction. J. Atmos. Sci., 71, 3788-3800, https://doi.org/ 10.1175/JAS-D-14-0067.1. 